\title{
4 Apoio matricial em saúde mental: uma análise sob ótica dos profissionais de saúde da atenção primária
}

Danielle Silveira da Silva ${ }^{1}$

\section{RESUMO}

\section{Objetivo}

Avaliar, sob a ótica dos profissionais da atenção primária, o apoio matricial como uma estratégia de cuidado em saúde mental, através da explanação das potências e limitações do apoio matricial realizado pelo Centro de Atenção Psicossocial - CAPS I Arthur Bispo do Rosário, da cidade de Aracaju/SE, nas Unidades Básicas de Saúde referenciadas: UBS João Cardoso de Melo, UBS Walter Cardoso, UBS Carlos Hardman, UBS Edézio Vieira de Melo e UBS João Oliveira Sobral.

\section{Métodos}

Trata-se de um estudo descritivo, exploratório com abordagem qualitativa realizada com profissionais da atenção primária presentes no momento da realização do apoio matricial. Utilizou-se um roteiro de entrevista semiestruturado composto de questões abertas sobre definição da proposta do apoio matricial, a forma de organização nos serviços, os possíveis obstáculos enfrentados e sugestões para melhoria do apoio matricial.

\section{Resultados}

Os profissionais configuraram de forma clara as potencialidades do apoio matricial no que diz respeito ao cuidado integral e compartilhado do usuário com transtorno mental e como a estratégia enriquece as possibilidades de composição interdisciplinar do Projeto Terapêutico Singular e desconstrói a lógica dos encaminhamentos intermináveis, melhorando o fluxo do usuário de transtorno mental na rede. Quanto aos obstáculos, foram apontados a deficiência de suporte nas unidades básicas de saúde, pouca aderência dos profissionais e a falta de capacitação permanente. Como alternativas para melhoria do apoio matricial foi sugerido pelos próprios profissionais a criação de espaços para aproximação com demais profissionais e capacitações para execução da Política de Saúde Mental.

\section{Considerações finais}

Apesar de incipiente, é de grande importância a formulação e o desenvolvimento de propostas de avaliação desta experiência, já que o apoio matricial se constitui uma estratégia privilegiada para transformações políticas e assistenciais da Saúde Mental.

\section{ABSTRACT}

\section{Objective}

To evaluate, from the perspective of primary care professionals, the support matrix as a strategy for mental health care, through a discussion of the strengths and limitations of the matrix support by the Center for Psychosocial Care - CAPS I Arthur Bispo do Rosário, city Aracaju / SE, in the Basic Health Units referenced: João Cardoso de Melo, Walter Cardoso, Carlos Hardman, Edézio Vieira de Melo e João Oliveira Sobral.

\section{Methods}

This is a descriptive, exploratory study conducted with a qualitative approach to primary care professionals present for the realization of the matrix support. The interview guide composed of semi-structured open questions about the proposed definition of the matrix support, the organization in the form of services, potential obstacles and suggestions for improving the support matrix.

\section{Results}

The professionals clearly shaped the potential of the matrix support with regard to comprehensive shared care user with a mental disorder and how the strategy enriches the possibilities of interdisciplinary composition of the Therapeutic Project Singular and deconstructs the logic of endless referrals improving the flow user's mental disorder on the network. The obstacles were mentioned disability support in primary health care, little adherence of professionals and lack of ongoing training. As alternatives to improve the support matrix has been suggested by professionals to create space for approach and skills with other professionals to implement the Mental Health Policy.

\section{Conclusion}

Although in its new, is of great importance to the formulation and development of proposals for evaluation of this experience, since the matrix support constitutes a privileged strategy for political change and care of mental health.

KEYWORDS: Matrix Support; Primary Attention; Care; Mental Health.

\section{PALAVRAS-CHAVE: Apoio Matricial; Atenção Primária;} Cuidado; Saúde Mental.

\footnotetext{
1 Enfermeira, Centro de Atenção Psicossocial - CAPS III Liberdade, danindia@hotmail.com Submetido em 11-08-2011. Aceite em 16-12-2011.

Citação: Silva, D. S. (2011) Apoio matricial em saúde mental: uma análise sob ótica dos profissionais de saúde da atenção primária. Revista Portuguesa de Enfermagem de Saúde Mental, 6, 20-27.
} 


\section{INTRODUÇÃO}

Este estudo pretende avaliar, sob a ótica dos profissionais da atenção primária, o Apoio Matricial como uma estratégia de cuidado em saúde mental, através da explanação das potências e limitações do Apoio Matricial realizado pelo Centro de Atenção Psicossocial - CAPS I Arthur Bispo do Rosário, da cidade de Aracaju/SE, nas Unidades Básicas de Saúde referenciadas.

De acordo com os princípios da reforma psiquiátrica, os hospitais psiquiátricos devem ser substituídos por uma rede composta por CAPS, ambulatórios de referência em saúde mental, residências terapêuticas, centros de convivência, emergências psiquiátricas em hospitais gerais e a assistência na atenção primária. Nos CAPS, os usuários com transtorno mentalsãoacompanhadosetratadosatravés depsicoterápicos, oficinas terapêuticas e grupos operacionais que atuam sobre a recuperação da saúde e reinserção social.

A atenção em saúde mental não atinge seus propósitos isoladamente, sendo viável a inclusão do principio da intersetorialidade. Pela razão da proximidade com as famílias e comunidade, as equipes da atenção primária devem ser consideradas estratégicas para garantir o acesso e o enfrentamento dos casos de saúde mental.

O Ministério da Saúde [MS] (2007) afirmara que a responsabilidade pelo cuidado aos usuários de saúde mental do território deve ser compartilhada entre as equipes de referência em saúde mental e equipes da atenção primária, excluindo a lógica do encaminhamento e visando aumentar a capacidade resolutiva de problemas de saúde pela equipe local.

O Apoio Matricial surgiu como arranjo de organização e de gestão dos serviços de saúde como forma de superar a racionalidade gerencial tradicionalmente verticalizada, compartimentalizada e produtora de processo de trabalho fragmentado e alienante para o trabalhador (MS, 2009).

Espera-se que o estudo possa contribuir com informações relevantes sobre o Apoio Matricial, a fim de sensibilizar os profissionais da saúde a desenvolverem ações direcionadas aos usuários com transtorno mental, pois estes também são clientes que necessitam do cuidado integral para garantir a qualidade de vida e poder reinserir-se na sociedade.

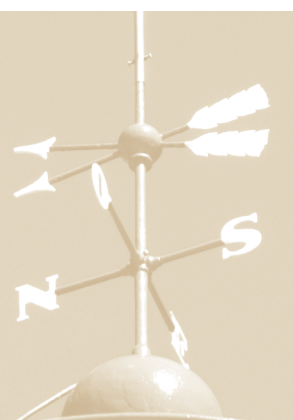

\section{REVISÃO DE LITERATURA}

O processo de desinstitucionalização da psiquiatria no Brasil avançou significadamente nos últimos tempos, expandiramse os serviços substitutivos aos hospitais psiquiátricos convencionais.

De acordo com o Relatório de Gestão 2003 a 2006 mudou-se o perfil da rede de hospitais psiquiátricos através do Programa Nacional de Avaliação do Sistema Hospitalar/PsiquiatriaPNASH/Psiquiatria- instituído em 2002 para avaliar sistematicamente e anualmente os hospitais psiquiátricos no Brasil- e do Programa Anual de Reenstruturação da Assistência Hospitalar Psiquiátrica no SUS- PRH- instituído em 2004, com estratégias de redução progressiva e pactuada dos leitos a partir dos macro-hospitais. (MS, 2007).

Para que a redução de leitos nos hospitais psiquiátricos ocorra de maneira planejada, de modo a garantir a continuidade da assistência ao usuário de transtorno mental, cria-se o Apoio Matricial em Saúde.

O Apoio Matricial surge como um modelo organizacional de trabalho, proposto inicialmente por Campos e, posteriormente, utilizada na gestão do cuidado na rede de saúde mental em Campinas /SP e adotada pelo MS através do Programa Humaniza-SUS, que pretende assegurar maior eficácia e eficiência ao trabalho em saúde mental e investir na construção de autonomia dos usuários da saúde mental do Sistema Único de Saúde- SUS (Campos, 2007).

A Política Nacional de Saúde Mental frisa que para a desistitucionalização é necessário que os serviços substitutivos- CAPS, ambulatórios de referência em saúde mental, residências terapêuticas, centros de convivência, emergências psiquiátricas em hospitais gerais e a assistência na atenção primária, se apresentem como uma rede que suporte a assistência a ser prestada.

Nessa perspectiva, o Apoio Matricial por ser um instrumento interlocutor do cuidado, facilita a intersetorialidade entre os serviços disponíveis da rede substitutiva do SUS. Campos e Domitti (2007) relatam que com o Apoio Matricialé possível ampliar as possibilidades de realizar-se a clínica ampliada e integração dialógica entre as distintas especialidades e profissões.

A coordenação de Saúde Mental, no documento apresentado à Conferência Regional de Reforma dos Serviços de Saúde Mental, define o apoio matricial como um arranjo organizacional que viabiliza o suporte técnico em áreas específicas para equipes responsáveis pelo desenvolvimento de ações básicas de saúde. Nesse arranjo, a equipe de saúde mental compartilha a corresponsabilização pelos a alguns casos com as equipes de Atenção Básica. Tal compartilhamento podem se efetivar através de discussões conjuntas de casos, intervenções conjuntas junto às famílias e comunidades ou em atendimentos conjuntos, e também na forma de supervisão e capacitação (MS, 2005). 
Para que haja a substituição da assistência psiquiátrica convencional e a garantia que os serviços dos SUS assegurem-se como substitutivos é indispensável à redução dos leitos em hospitais psiquiátricos e/ou fechamento destes, como também o reforço ao fortalecimento da saúde mental na atenção primária.

Segundo estimativas internacionais e do MS 3\% da população, ou seja, cinco milhões de pessoas são casos de transtornos mentais graves e crônicos, sendo $9 \%$ desde montante os casos de transtornos mentais moderados a leves e 6 a $8 \%$ decorrentes ao uso abusivo de álcool e outras drogas (MS, 2003).

O MS (2003) publica que $56 \%$ das equipes da saúde da família realizam ações de saúde mental. Tais estatísticas demonstram que a atenção primária tem uma valiosa importância na assistência às demandas psiquiátricas, o que torna justificável a realização do Apoio Matricial.

Os participantes do Apoio Matricial são os profissionais das diversas áreas especializadas tanto do CAPS, quanto da atenção primária e da assistência social, respeitando a lógica que a equipe multidisciplinar de saúde amplia o olhar no campo de atuação e qualifica a assistência ao usuário com transtorno mental.

Essa nova estratégia de cuidado em saúde mental modifica a forma de operacionalizar a referência e contra-referência dos serviços de saúde, ou seja, a responsabilidade do cuidado ao usuário e de sua família será compartilhada entre a rede primária e a rede especializada em saúde mental. Por conseguinte, os encaminhamentos são reduzidos, devido à facilitação do contato direto entre os serviços.

Campos e Domitti (2007) referem que há duas maneiras para realização do Apoio Matricial. Primeiro, mediante a combinação de encontros periódicos e regulares, a cada semana, quinzena ou mais espaçados. Nesses encontros, objetiva-sediscutircasosou problemasdesaúdeselecionados pela equipe de referência e procura- se elaborar Projetos Terapêuticos Singular- PTS e acordar linhas de intervenção entre os profissionais envolvidos. Segundo, em casos imprevistos e urgentes, em que não seria recomendável aguardar a reunião regular, o profissional de referência aciona o Apoio Matricial por contato pessoal, eletrônico ou telefônico e não apenas por meio de encaminhamento impresso entregue ao paciente.

Além de ser um regulador de fluxo e favorecer a corresponsabilização do cuidado, o Apoio Matricial permite diferenciar os casos que precisam ser atendidos nos serviços especializados e os que podem ser acompanhados pela equipe saúde da família, ou pelo menos ser acolhidos momentaneamente por esses profissionais (Bezerra \& Dimenstein, 2008).

Para nortear o cuidado ofertado no Apoio Matricial, o PTS é uma ferramenta baseada nas necessidades individuais de cada usuário e de sua família, que utiliza os serviços comunitários e os serviços de saúde disponíveis pelo SUS. O MS (2009) define o PTS como um conjunto de propostas de condutas terapêuticas articuladas, para um sujeito individual ou coletivo, resultado da discussão coletiva de uma equipe interdisciplinar.

Campos e Domitti (2007) descrevem que a construção do PTS pode se desenvolver em três planos fundamentais: atendimentos e intervenções conjuntas; atendimentos e intervenções especializadas, contanto, com cuidado complementar da outro serviço de saúde; troca de conhecimentos e de orientações entre os serviços através de diálogos sobre alterações na avaliação do caso e mesmo reorientação das condutas antes adotadas, permanecendo, o caso sob cuidado da referência.

Para viabilizar a construção do PTS, o Apoio Matricial seria um dispositivo valioso, o MS (2009) ressalta que todas as opiniões das equipes são importantes para ajudar a entender o sujeito com alguma demanda de cuidado em saúde e, consequentemente, para definição de propostas de ações.

Por ser o Apoio Matricial uma nova estratégia de cuidado em saúde mental pactuada por profissionais e gestores pressupõe que existam formas peculiares de sua configuração a depender dos recursos disponíveis de cada região. O que não exime a possibilidade de existência de obstáculos enfrentados no processo de construção e organização pelas equipes de saúde. Portanto, o Apoio Matricial precisa ser avaliado com constância para evitar que os impasses enfraqueçam o modo de operá-lo.

\section{MATERIAL E METODOS}

O método utilizado para a realização foi o descritivo, exploratório e participativo com abordagem qualitativa. $\mathrm{O}$ universo da pesquisa foi na cidade de Aracaju, capital de Sergipe. Como unidades de exploração foram escolhidas as UBS referenciadas pelos CAPS I Arthur Bispo do Rosário, que são: UBS João Cardoso de Melo, UBS Walter Cardoso, UBS Carlos Hardman, UBS Edézio Vieira de Melo, UBS João Oliveira Sobral.

O CAPS I Arthur Bispo do Rosário criado em 2000, pertence à Associação Luz do Sol, órgão não governamental, vinculado à Secretaria Municipal de Saúde de Aracaju. Seu território abrangência são os bairros Santos Dumont, Soledade, Siqueira Campos, José Conrado de Araújo e Veneza.

As UBS pesquisadas são compostas por 02 a 04 equipes multiprofissionais, sendo a conformação básica formada por médicos, enfermeiros, auxiliares de enfermagem, agentes comunitários de saúde, dentista, auxiliar de consultório dentário e assistente social. A UBS Edézio Vieira de Melo tem equipe ampliada e é ambulatório de referência em 
saúde mental, na sua conformação ainda há o psicólogo e psiquiatra.

A coleta de dados foi autorizada mediante aprovação do Comitê de Educação Permanente da Secretaria Municipal de Saúde - SMS de Aracaju, já que se tratava de uma pesquisa institucional. E subseqüentes Coordenação da Rede de Atenção Psicossocial- REAPS, Núcleo em Gestão de Pessoas- NUGEP da SMS de Aracaju e coordenações do CAPS I Arthur Bispo do Rosário e das UBS referenciadas.

Foram entrevistados os profissionais das UBS referenciadas pelo CAPS I Arthur Bispo do Rosário que assinaram o Termo de Consentimento Livre Esclarecido- TCLE e que se faziam presentes no momento do Apoio Matricial. Utilizouse um roteiro de entrevista semi-estruturado composto de questões abertas sobre a definição da proposta do apoio matricial, a forma de organização nos serviços, os obstáculos enfrentados e possíveis sugestões para melhoria deste.

Foram entrevistados um total de 15 profissionais, distribuídos entre as seguintes categorias profissionais: 01 psicólogo, 03 assistentes sociais, 03 enfermeiras, 08 agentes comunitários de saúde.

\section{RESULTADOS E DISCUSSÃO}

A fim de sintetizar os resultados e discussão serão apresentados os principais achados das narrativas construídas pelos entrevistados. Foram separados pelos tópicos: Definição da Proposta do Apoio Matricial, Organização do Apoio Matricial, Obstáculos do Apoio Matricial e Alternativas para Melhoria do Apoio Matricial. Buscou-se identificar através de seus elos os principais pontos de potencialidade e de limitações que a estratégia suscita.

\section{DEFINIÇÃO DA PROPOSTA DO APOIO MATRICIAL}

Em relação à definição do Apoio Matricial, a maioria dos entrevistados soube definir de forma clara o significado do Apoio Matricial, avaliando como positiva a presença do profissional do CAPS I Arthur Bispo do Rosário. As definições se concentraram em um espaço de trocas de experiências e de saberes no campo da saúde mental com outros profissionais da atenção primária, sendo uma construção coletiva que favorece a corresponsabilidade e a avaliação continua no tratamento do usuário. Os principais tópicos que definiram a proposta do apoio matricial foram:

"Encontro entre as partes envolvidas no cuidado em saúde mental do território, no caso CAPS e ambulatório de referencia, para discutir os casos em comum, busca de soluções e de encaminhamentos para tratamento dos usuários." (Psicólogo 1)
"Discussão dos casos de saúde mental entre os profissionais da USF e CAPS e orientações quanto às intervenções nos casos mais difíceis de solucionar." (Assistente Social 1)

"É o apoio que uma equipe de saúde mental multidiciplinar dá à equipe de saúde das Unidades Básicas, monitorando os casos de pacientes com alguns tipos de transtornos mentais, seja por álcool e drogas ou não." (Agente Comunitário de Saúde 5a)

A proposta do Apoio Matricial, segundo Campos e Domitti (2007), aponta exatamente a construção de estratégias integradas entre as equipes que permite a construção coletiva de praticas de intervenção, explorando os diversos saberes sobre os casos apresentados, privilegiando a corresponsabilidade.

Os autores supracitados reforçam a importância do Apoio Matricial ao destacar como uma metodologia de trabalho que visa assegurar a retaguarda especializada, tanto em nível assistencial quanto técnico pedagógico, pressupondo uma construção compartilhada entre a equipe de referência e os apoiadores que visa aumentar a capacidade resolutiva dos problemas.

Outra potencialidade do Apoio Matricial em saúde mental, conforme será apresentado pela citação a seguir, demonstra uma modificação do foco do cuidado, que se constrói a partir das necessidades dos indivíduos, das famílias e do meio social. Dessa maneira, o PTS também se beneficia ganhando mais significância.

"São reuniões para melhorar a situação de cada paciente e também oferecer o cuidado a família." (Agente Comunitária de Saúde 4a)

\section{ORGANIZAÇÃO DO APOIO MATRICIAL}

O Apoio Matricial nas unidades pesquisadas possui a mesma freqüência de realização. Os profissionais se encontram mensalmente, cuja data é escolhida por conveniência dos horários, sendo fixa para os meses posteriores. Como explanam os entrevistados:

"O apoio é organizado mensalmente, através de uma agenda fixa, as primeiras quartas-feiras do mês." (Agente Comunitário de Saúde 4b)

"Temos nos reunidos uma vez por mês, discutindo os casos e dando os encaminhamentos. No momento estamos procurando envolver mais profissionais neste cuidado que tem basicamente a participação de três funcionarias." (Psicólogo 1)

Apesar de haver datas e horários previstos para os encontros, ainda há restrição na participação de outros 
profissionais nos encontros, o que concebe a um desvio de responsabilidade do cuidado. Não se trata apenas pela fragmentação imposta pelo processo de trabalho, na obrigação da realização das Ações Programáticas de Saúde, mas também de outras razões que valem ser exploradas. Um fato preocupante, já que o Apoio Matricial deve ser discutido por todos os envolvidos, a fim de aumentar a eficácia das intervenções.

Para Campos (1999) o reconhecimento da necessidade de eventual comunicação entre as distintas profissões representa um avanço, um início de enfrentamento do problema, mas apenas com discurso não é alterada a estrutura que reforça a esta separação. Na lógica da integração episódica, eventuais encontros horizontais tentariam amainar os conflitos e desacertos.

Além de discussões de casos, os profissionais aproveitam a oportunidade do encontro para realizar visitas domiciliares e consultas multidisciplinares aos possíveis e confirmados casos de transtorno mental. Tal ação propõe a integração entre a equipe da atenção primária com os profissionais da saúde mental. Através das visitas ocorre a desconstrução do esquema tradicional de encaminhamentos e das guias de referência e contra referência, além de compor o Projeto Terapêutico Singular. Conforme apontado nos depoimentos:

"Nos reunirmos uma vez ao mês para discutir sobre os pacientes e, se necessário, fora deste dia, ligamos e agendamos visitas, consultas e etc." ( Enfermeiro 2)

"Todo o mês o CAPS tem a responsabilidade de comparecer na unidade para realizar o apoio matricial, inclusive fazemos visitas conjuntas a usuários que seja caso de doença mental e compomos o plano de cuidados." (Assistente Social 3)

Campos (1999) propôs um rearranjo organizacional do apoio matricial, sugeriu reformas estruturais na linha de cuidado para produzir saúde com um grau maior de resolutividade e desalienar os profissionais de saúde em relação ao objetivo único de seu trabalho. Logo, o acompanhamento longitudinal dos usuários passa a ser horizontal, estimulando o vínculo e a uma atenção compromissada dos profissionais com os usuários de transtorno mental.

A relação terapêutica, horizontal, passa ser reguladora do processo de trabalho. Assim, toda vez que o usuário necessitar do serviço, é atendido pela sua equipe e seu profissional de referencia, o que permite um acompanhamento do processo saúde/doença/intervenção de cada usuário (Campos, 1999).

A partir das discussões clínicas e intervenções conjuntas, como consultas, visitas domiciliares, entre outras, os profissionais de saúde contribuem para aumento da capacidade resolutiva dos casos, qualificando para uma atenção ampliada em saúde que contemple as necessidades dos usuários e das famílias. Dessa forma o Projeto
Terapêutico Singular acaba sendo construído de uma maneira não protocolada, de acordo com a vulnerabilidade e potencialidade de cada usuário.

\section{OBSTÁCULOS DO APOIO MATRICIAL}

Existe uma série de obstáculos apontados pelos profissionais que precisam ser reconhecidos, analisados e, se possíveis solucionados. Dentre eles, a deficiência de suporte nas unidades, o número insuficiente de profissionais que participam do Apoio Matricial e a própria maneira como os serviços vêm se estruturando para a realização do cuidado. Todavia, não se coloca como um entrave definitivo, desde que todos os profissionais tenham orientações e suporte técnico adequados.

"Penso que na prática ainda temos o desafio de envolver um quantitativo dos profissionais da unidade, a dificuldade parece ser a compreensão de que a saúde mental faz parte do cuidado." (Psicólogo 1)

"A falta de compromisso dos profissionais e da gestão em não proporcionar espaços para que todos se façam presentes." (Assistente Social 3)

Apesar das UBS pesquisadas possuírem uma composição multiprofissional, a comunicação e integração entre as distintas especialidades e profissão não estavam em coesas. Consoante ao depoimento:

"Acredito que o horário disponível dos profissionais das equipes PSF, seja difícil a conciliação, devido às várias atribuições. À exemplo dos médicos que nunca podem participar pela falta de disponibilidade." ( Assistente Social 1)

Observa-se que o Apoio Matricial ainda não é uma estratégia de cuidado consolidada, e mesmo com o comprometimento da equipe de referência, há um alto grau de dependência do compartilhamento dos demais saberes. Esse cenário favorece aos incômodos gerenciais e constitui-se em outro impasse à adoção do método de trabalho do Apoio Matricial.

Em medicina e na saúde em geral houve uma crescente divisão do trabalho que dificulta a integração do processo de atenção e cuidado as pessoa, já que as distintas especialidades médicas e as profissões de saúde definiram objetos de intervenção e campos de conhecimento sem grandes compromissos com a abordagem integral de processos de saúde e doença concretos (Camargo, 2003).

Soma-se ainda que o médico seja visto como o profissional mais eficiente para prestar os cuidados, tendo então os profissionais da referência de saúde mental a árdua tarefa de desmistificar tal conceito através da incorporação do princípio da integralidade do cuidado, na perspectiva que a 
participação da equipe multiprofissional, independente do nível de escolaridade, é peça essencial para o cuidado.

Apesar da grande maioria dos profissionais entrevistados conhecerem seu papel no encontro, foi perceptível em duas UBS que alguns poucos profissionais presentes durante o Apoio Matricial não compreendiam a definição e as finalidades deste, configurando-se também como um obstáculo. Conforme demonstrado pelos seguintes depoimentos:

"O maior obstáculo é que às vezes não conseguimos nos reunir e também não sentimos a resolutividade de alguns casos, mas não sei por qual o motivo." (Enfermeiro 2)

"Um maior esclarecimento do papel do apoio matricial." (Agente Comunitário de Saúde 5a)

Campos (2003) propõem que na qualificação das equipes da atenção primária seja incorporado outras dimensões do sujeito além de sua faceta biológica, valorizando a subjetividade e o conjunto das relações sociais que determinam desejos, interesses e necessidades. Ao mesmo momento que a equipe esteja acompanhada por um especialista da referência que lhes dê suporte para compreender e intervir nessa prática.

Outro empecilho que inviabiliza o Apoio Matricial apontado por todas as UBS foi a falta de um transporte para realização de visitas, encaminhamentos de usuários ao CAPS e encontro com outros profissionais, já que não há disponível um transporte para cada UBS no município de Aracaju, sendo necessário agendamento com antecedência junto a Secretaria Municipal de Saúde. Como as descrições abaixo:

"Dificuldade de transporte para visitar os usuários." (Enfermeiro 3)

"As barreiras colocadas devido a dificuldade para levar pacientes aos CAPS." (Agente Comunitário de Saúde 4b)

"O obstáculo é do deslocamento das pessoas." (Agente Comunitário de Saúde 5b)

\section{ALTERNATIVAS PARA MELHORIA DO APOIO MATRICIAL}

A partir da descrição dos obstáculos, os quais esbarram a implantação do Apoio Matricial, o estudo teve a intenção de estimular a capacidade de intervenção dos entrevistados em levantar proposições e buscar alternativas para superar tais impasses. Os profissionais constataram a necessidade de qualificação, através da criação de espaços de capacitações permanentes, que propiciem a sensibilização, além de, expor suas dúvidas e angústias. Conforme os relatos:
"Penso que uma roda de conversa entre os profissionais, questionando suas dúvidas e inquietações e também promovendo o aparecimento do "Know How" de quem já participa. Seria um bom incremento para o processo de assimilação do fazer de cada um.” (Psicólogo 1)

"Sensibilizar os profissionais para importância do trabalho desenvolvido e as ações do Programa de Saúde Mental." (Assistente Social 1)

"Qualificar a equipe dos serviços de assistência e retaguarda, agenda positiva de ações entre os serviços da rede." (Assistente Social 5).

Figueiredo (2006) destaca a capacitação in loco como fundamental para que as equipes aprendam, durante a prática, a intervir no campo da subjetividade. Atenta que na tentativa de qualificar a assistência, roteiros são criados a fim de aprimorar a coleta de informações e a orientar os atendimentos. Reforça, entretanto, o cuidado para que eles não se transformem em scripts ou manuais de passo a passo, provocando trabalho alienado e burocratizando os atendimentos.

Por consenso, todos os profissionais indicam a necessidade de novas estratégias para incrementar a participação de demais membros das equipes no Apoio Matricial. Nesse sentido, surge um grande ponto de tensão que explicita certa imprecisão das fronteiras entre o que é de responsabilidade de quem. Alguns profissionais consideram especialmente a função de sustentação e articulação dos gestores com as equipes, para que as reuniões do Apoio Matricial façam parte da rotina de trabalho. Por outro lado, outros apostam que a melhoria depende da responsabilidade e da disponibilidade dos próprios profissionais.

"Maior cobrança da coordenação do serviço. Tem que haver certa cobrança aos profissionais." (Assistente Social 3)

"Acredito que deveria haver um maior posicionamento da gestora para manter o matriciamento e melhoria no transporte para realizar o cuidado." (Enfermeira 2)

"Para melhoria do cuidado aos usuários de transtorno mental deveria ter o compromisso e responsabilidade de toda a equipe, inclusive da gestão. E para continuidade do apoio matricial, as visitas poderiam ser agendada com antecedência para que o transporte fosse assegurado pela Secretaria de Saúde." (Enfermeira 3)

"Uma maior disponibilidade de tempo dos profissionais para um melhor acompanhamento dos casos." (Agente Comunitário de Saúde 5a)

Diante do exposto, é inegável a necessidade de um suporte para nortear a assistência ao usuário de saúde mental nas UBS referenciadas pelo CAPS I Arthur Bispo do Rosário, com a oferta de capacitações e treinamentos contínuos. A 
promoção de encontros com a gestão, equipes de referência e equipes da atenção primária proporcionam uma atuação mais integral e menos fragmentada, em que o maior beneficiado é o usuário.

\section{CONSIDERAÇÕES FINAIS}

O Apoio Matricial como estratégia recente de cuidado ainda perpassa por um processo de construção e renovação. Muitos são os avanços em desenvolvimento e outras tantas são os obstáculos reconhecidos. A qualificação do Apoio Matricial é um grande desafio para a gestão da Saúde Mental. O fortalecimento de uma política mais efetiva de formação continuada para os profissionais da atenção primária deverá ser uma das prioridades da Política de Saúde Mental.

É inegável a potencialidade do Apoio Matricial no que diz respeito ao cuidado integral e compartilhado. A estratégia enriquece as possibilidades de composição interdisciplinar do Projeto Terapêutico Singular e desconstrói a lógica dos encaminhamentos intermináveis, melhorando o fluxo do usuário de transtorno mental na rede.

Os profissionais envolvidos no apoio matricial assumem o papel de apoiadores, acolhem e orientam os usuários na perspectiva holística. Todavia a pouca aderência dos profissionais envolvidos nesse processo, a falta de capacitações permanentes e de estrutura se configuram como obstáculos que emperram a operacionalização do Apoio Matricial.

Foi constatado que há uma necessidade emergencial de sensibilização e capacitação de todos os profissionais que atuam nas UBS referencias pelo CAPS I Arthur Bispo do Rosário para o atendimento à demanda de casos de saúde mental. Com isso, possivelmente, o Apoio Matricial se fortaleça e seja processado de maneira mais clara pelas equipes para que os profissionais não o vejam como um trabalho a mais.

Para sensibilizar os profissionais da atenção primária temse como sugestão a formação de rodas de conversas para reflexão e discussão da Política de Saúde Mental; formulação e apresentação de propostas de roteiro de orientações para atendimento a demanda de casos de saúde mental (Anexo A); discussão dos casos de saúde mental nas reuniões de equipes e mini equipes; formação de novas parcerias intersetoriais através do conhecimento dos demais dispositivos das redes e das comunidades; socialização dos saberes para elaboração do PTS e educação permanente para os profissionais e gestores.

É imprescindível informar que não se deve somente atribuir as responsabilidades para o outro, haja vista a necessidade dos profissionais de saúde repensar na lógica de operacionalizar - Apoio Matricial. Com a ciência da significância na participação nesse processo, os profissionais contribuirão para uma realidade diferente, onde o cuidado integral e o trabalho multiprofissional são as peças transformadoras.

\section{REFERÊNCIAS BIBLIOGRÁFICAS}

Bezerra, E., \& Dimenstein, M. (2008). O CAPS e o Trabalho em Rede: Tecendo o Apoio Matricial na Atenção Básica. Psicologia, Ciência e Profissão, 28(3), 632-645.

Camargo, Jr. K. R. (2003). Biomedicina, Saber \& Ciência: uma abordagem crítica. São Paulo, SP: Hucitec.

Campos, G. W. de S. (1999). Equipes de referência e apoio especializado matricial: um ensaio sobre a reorganização do trabalho em saúde. Ciência e Saúde Coletiva, 4 (2), 393403 .

Campos, G. W. de S. (2003). A clínica do sujeito: por uma clínica reformulada e ampliada. São Paulo, SP: Hucitec.

Campos, G. W. de S., \& Domitti, A. C. (2007). Apoio matricial e equipe de referência: uma metodologia para gestão do trabalho interdisciplinar em saúde. Cadernos de Saúde Pública, 23 (2), 399-407.

Figueiredo, M. D. (2006). Saúde Mental na Atenção Básica: Um estudo Hermenêutico-Narrativo sobre o Apoio Matricial na Rede SUS-Campinas (SP). Dissertação de mestrado não publicada, Universidade Estadual de Campinas, São Paulo, Brasil.

Ministério da Saúde (2000). Relatório de Actividades Rede Nacional de Escolas Promotoras de Saúde. Lisboa: Ministério da Saúde / Direcção Geral da Saúde / Divisão de Saúde Escolar.

Ministério da Saúde. (2003). Saúde Mental e Atenção Básica: o vinculo e o diálogo necessários. Brasília: Coordenação de Saúde Mental/ Coordenação de Gestão da Atenção Básica.

Ministério da Saúde. (2005). Reforma Psiquiátrica e Política de Saúde Mental no Brasil. Brasília: Secretaria de Atenção à Saúde/DAPE. Coordenação Geral de Saúde Mental.DF.

Ministério da Saúde. (2007). Relatório de Gestão 2003-2006. Saúde Mental no SUS: acesso ao tratamento e mudança do modelo de atenção. Brasília: Secretaria de Atenção à Saúde/DAPE.

Ministério da Saúde. (2009). Clínica Ampliada e Compartilhada. Brasília: Secretaria de Atenção à Saúde/ Política Nacional de Humanização da Atenção e Gestão do SUS. Textos Básicos de Saúde. 
Ministério da Saúde. (2011). Relatório de Gestão 2007-2010. Saúde Mental no SUS: as novas fronteiras da Reforma Psiquiátrica. Brasília: Secretaria de Atenção à Saúde/ DAPES. Coordenação Geral de Saúde Mental, Álcool e Outras Drogas. 